\title{
Contracepção hormonal: uma comparação entre pacientes das redes pública e privada de saúde
}

\author{
Hormonal contraception: a comparison \\ between patients of the private and public health network
}

Patrícia Delage Gomes ${ }^{1}$

Juliana Barroso Zimmermmann ${ }^{1}$

Lizandra Maris Borges de Oliveira ${ }^{1}$

Kátia Aureana Leal ${ }^{1}$

Natália Delage Gomes ${ }^{1}$

Soraia Moura Goulart ${ }^{1}$

Dilermando Fazzito Rezende ${ }^{1}$
${ }^{1}$ Faculdade de Medicina de Barbacena, Fundação José Bonifácio Lafayette de Andrada. Praça Presidente Antonio Carlos 8. 36202-336 Barbacena MG natdelage@hotmail.com

\begin{abstract}
The aim of this paper is to assess the profile of patients using hormonal contraceptives in the public health network and a comparison with the private health service, as well as the frequency of side effects and adherence to treatment. A cross-sectional study was conducted with 240 patients, namely 120 patients from the private health service and 120 patients from the public health network. The most commonly prescribed hormonal dosage on the private group (36.7\%) was 15 or 20 micrograms of ethinyl estradiol (EE), associated with gestodene, desogestrel or levonorgestrel. On the other hand, the prescribed hormonal dosage in the public group was a combination of 30 micrograms of EE associated with gestodene, levonorgestrel or desogestrel (48.3\%). There was no difference between the frequency of side effects in both groups surveyed ( $p>0.05)$. Meanwhile, adherence to treatment was higher in patients of the private group. The authors concluded that the most widely used contraceptive method was a low oral dose of ethinyl estradiol and there is no difference between the frequency of side effects. However, adherence to treatment was higher in the private group, which may be associated with social and cultural aspects of the patients surveyed.
\end{abstract}

Key words Contraception, Prescription, Adherence
Resumo O objetivo deste artigo é avaliar o perfil de pacientes usuárias de contraceptivos hormonais no setor público e fazer a comparação com o serviço privado de saúde, bem como verificar a frequência de efeitos colaterais e a aderência ao tratamento. Realizou-se um estudo de corte transversal com 240 pacientes, sendo 120 da rede privada e 120 da rede pública de saúde. No grupo privado, a dosagem hormonal mais frequentemente prescrita foi a de 15 ou 20 microgramas de etinil-estradiol (EE), associados a gestodeno, desogestrel ou levonogestrel (36,7\%). No grupo público, a combinação de 30 microgramas de EE associados a gestodeno, levonogestrel ou desogestrel (48,3\%) foi a principal medicação contraceptiva prescrita. Não houve diferença entre a frequência dos efeitos colaterais nos dois grupos pesquisados ( $p>0,05)$. A aderência ao tratamento foi maior nas pacientes do grupo privado $(p<0,05)$. Concluiu-se que o método contraceptivo mais utilizado foi o oral com baixa dosagem de EE, não havendo diferença entre a frequência dos efeitos colaterais. A aderência ao tratamento foi maior no grupo privado, o que pode estar associado ao fator sociocultural das pacientes estudadas.

Palavras-chave Contracepção, Indicação, Aderência 


\section{Introdução}

Desde a sua introdução, os contraceptivos hormonais representam a forma mais utilizada de anticoncepção em todo o mundo. A evolução contínua dos contraceptivos hormonais apresenta características expressivas, já que a primeira formulação oral continha doses elevadas de etinilestradiol $(150 \mathrm{mcg})$ e de noretinodrel $(9,85$ $\mathrm{mg})^{1}$, associando-se a complicações cardiovasculares (tromboembolismo venoso, infarto agudo do miocárdio, acidente vascular cerebral $)^{2,3}$. Estabeleceu-se relação entre a elevada dose de estrogênio e o tromboembolismo venoso e ao progestágeno, o prostágeno e os eventos cardiovasculares arteriais ${ }^{2}$.

Com a redução dessas dosagens, houve sensível diminuição do risco cardiovascular, e com a descoberta dos novos progestágenos, introduziu-se no mercado compostos com elevada tolerabilidade e eficácia contraceptiva ${ }^{2,3}$. Além disso, outras vias para a administração da medicação hormonal foram desenvolvidas. A via transdérmica, transvaginal e intrauterina minimizaram os efeitos gastrointestinais e auxiliaram na manutenção de um nível hormonal contínuo, impedindo a oscilação do nível hormonal, o que pode acontecer com o esquecimento da medicação quando utilizada por via oral ${ }^{4}$.

$\mathrm{O}$ fato é que a mulher moderna deseja muito mais dessas medicações do que o benefício inequívoco da contracepção. O controle da pele, com redução das acnes, o controle do ciclo menstrual, a redução das cólicas menstruais e da tensão pré-menstrual (TPM) são exigências da mulher que podem ser perfeitamente atendidas quando a prescrição é individualizada. Além disso, muitas mulheres sequer desejam menstruar ciclicamente, e já se considera a utilização da contracepção contínua, atendendo à melhoria da qualidade de vida das pacientes 5 .

Entretanto, acreditamos que esses benefícios adicionais não são obtidos por todas as pacientes. Algumas não conseguem obter o benefício da contracepção, porque não sabem utilizar a medicação adequadamente, não tem acesso ao sistema de saúde nem à medicação. Além disso, a prescrição raramente é individualizada, o que pode estar associado ao aumento dos efeitos colaterais $\mathrm{e}$, consequentemente, à suspensão da medicação. O abandono da contracepção expõe a mulher à gravidez indesejada, assim como contribui para a piora do nível socioeconômico do país.

Assim, nos propomos avaliar os aspectos clínicos e epidemiológicos de pacientes usuárias de contracepção hormonal nas redes pública e privada, bem como os principais efeitos colaterais identificados, a tolerabilidade e a aderência ao uso da medicação contraceptiva prescrita.

\section{Metodologia}

A presente investigação é um estudo de corte transversal realizado com pacientes usuárias de contracepção hormonal, no período de janeiro de 2001 a dezembro de 2006, tendo sido aprovado pelo Comitê de Ética e Pesquisa da Universidade presidente Antonio Carlos (Unipac).

$\mathrm{O}$ estudo constou de uma avaliação sobre a indicação da contracepção hormonal, efeitos colaterais e aderência ao tratamento em pacientes atendidas na rede privada (clínica particular) e rede pública de saúde (Faculdade de Medicina de Barbacena - Unidade Básica de Saúde Santa Cecília).

Todas as pacientes foram atendidas por um mesmo profissional e estiveram em pelo menos duas consultas realizadas com intervalos de três a seis meses entre elas. As informações contidas nos prontuários e utilizadas no presente estudo foram relacionadas aos aspectos clínicos (motivo da consulta, irregularidade menstrual, acnes, hirsutismo, paridade, dismenorreia, tensão prémenstrual, uso de contracepção prévia e possíveis efeitos colaterais), doenças preexistentes (hipertensão grave, diabetes mellitus com comprometimento vascular, câncer de mama, trombose venosa), cirurgias prévias e outros tratamentos anteriores. Além disso, foram anotados os dados referentes ao exame ginecológico e à prescrição contraceptiva escolhida, que obedeceu aos critérios de elegibilidade da Organização Mundial da Saúde (OMS) ${ }^{6}$, bem como dados referentes ao controle do uso dessa medicação, presença ou ausência de efeitos colaterais (menores: cefaleia discreta, enjoo, sangramento irregular, mudanças de humor e sensibilidade nas mamas; maiores: dor no abdome e no tórax persistentes, icterícia, cefaleias persistentes/severas, perda breve da visão e trombose venosa profunda), além de dados da avaliação clínica das pacientes.

$\mathrm{Na}$ análise dos dados, as informações obtidas dos prontuários foram transcritas por digitação para meio magnético e processadas em computador através de recursos de processamento estatístico do software EpiInfo, versão 6.04. Foram construídas as distribuições de frequência das variáveis examinadas e calculadas as taxas de prevalência indicadas para cada caso e também calculados as médias e os desvios pa- 
drão de variáveis expressas em escala numérica. A comparação das características das pacientes do setor público com as do setor privado foi realizada em tabelas de contingência, tipo $\mathrm{R} x \mathrm{C}$, no caso de variáveis categóricas, ou através da comparação de médias, no caso de variáveis numéricas. No teste de significância estatística das diferenças observadas na análise, utilizou-se o teste do Qui-quadrado $\left(\chi^{2}\right)$ e/ou o teste de Student $(\mathrm{T})$, dependendo da natureza dos dados comparados. O nível de significância adotado na análise foi de $5 \%$.

\section{Resultados}

\section{Características da amostra}

As pacientes são jovens (idade variando entre 18 e 24 anos) e com vida sexual ativa, sendo que as pacientes da rede pública de saúde tiveram maior número de gestações e partos quando comparadas com as da rede privada $(\mathrm{p}<0,05)$.

Quando se avaliou a profissão, verificou-se que as pacientes da rede pública trabalham preferencialmente no lar, enquanto as pacientes da rede privada de saúde são, em geral, estudantes ou trabalham fora do lar $(\mathrm{p}<0,05)$.

O exame de rotina, corrimento vaginal, contracepção, irregularidade menstrual, tensão prémenstrual (TPM) e as manifestações androgênicas foram os mais frequentes motivadores de consultas. Quando se compararam os dois grupos, verificou-se que as manifestações androgênicas $(p=0,0002)$, TPM $(p=0,012)$, corrimento vaginal $(p=0,012)$ e contracepção $(p=0,003)$ foram os motivadores de consultas associados às pacientes da rede privada de saúde $(\mathrm{p}<0,05)$. O exame de rotina $(\mathrm{p}=0,39)$, a irregularidade menstrual ( $\mathrm{p}=0,22)$ e dor pélvica $(\mathrm{p}=0,46)$ foram queixas frequentes, com distribuição semelhante nos dois grupos estudados $(\mathrm{p}>0,05)$.

Não se verificou diferença em relação ao exame das mamas $(\mathrm{p}=0,68)$, avaliação da pressão arterial $(p=0,69)$, da genitália externa $(p=0,20)$, trofismo vaginal $(\mathrm{p}=0,50)$ e fluxo genital $(\mathrm{p}=0,22)$. Entretanto, verificou-se diferença significativa em relação à presença de acnes $(\mathrm{p}=0,0003)$, abdome doloroso à palpação $(\mathrm{p}=0,0018)$ e abdome globoso $(\mathrm{p}=0,022)$, que foram associados às pacientes da rede privada de saúde. Essas pacientes apresentaram sobrepeso e obesidade com maior frequência $(7,5 \%)$, quando comparadas com as pacientes da rede pública $(2,7 \%)$, mas estes resultados não foram significativos $(p=0,12)$. $O$ mesmo pôde-se afirmar em relação à distribuição de pelos $(\mathrm{p}=0,27)$.

As infecções genitais mais frequentes nos dois grupos foram a candidíase, a tricomoníase e a vaginose bacteriana. Embora o fluxo genital patológico tenha sido motivador de consultas em 3,0\% das pacientes da rede pública, o exame ginecológico identificou o mesmo em $27,7 \%$. A Tabela 1 mostra os principais dados comportamentais, clínicos e gineco-obstétricos das pacientes estudadas.

\section{A prescrição contraceptiva}

Os principais motivadores da prescrição do contraceptivo hormonal, além do objetivo básico (contracepção), foram: tratamento da manifestação androgênica, irregularidade do ciclo menstrual, dismenorreia e TPM. Quando se compararam os dois grupos de pacientes (rede pública $\mathrm{x}$ priva$\mathrm{da})$, verificou-se que os motivadores associados às pacientes da rede privada de saúde foram o tratamento de manifestação androgênica $(\mathrm{p}=0,02)$, o controle da dismenorreia $(\mathrm{p}=0,01)$, TPM e controle da retenção hídrica $(\mathrm{p}=0,04)$. Por sua vez, o controle do ciclo menstrual e o da lactação foram indicações semelhantes nos dois grupos $(p>0,05)$.

A escolha da medicação contraceptiva obedeceu aos critérios de elegibilidade da Organização Mundial da Saúde (OMS). O contraceptivo hormonal oral foi o mais prescrito na rede pública de saúde, em dosagem de $30 \mathrm{mcg}$ de etinilestradiol (EE) (48,3\%), já que a disponibilidade da medicação no posto de saúde foi critério importante na escolha da dosagem $(\mathrm{p}<0,05) . \mathrm{Na}$ rede privada de saúde, o contraceptivo hormonal oral foi o mais prescrito $(36,7 \%)$, em dosagem de 15 ou 20 mcg de $\operatorname{EE}(\mathrm{p}<0,05)$. Neste grupo, observou-se uma contracepção individualizada, obedecendo a critérios clínicos específicos, como por exemplo tratamento das manifestações androgênicas $(\mathrm{p}=0,01 \sim \mathrm{OR}=0,38 \sim \mathrm{IC}=0,18$ $0,84)$, tratamento da TPM e correção da retenção hídrica ( $\mathrm{p}=0,000 \sim \mathrm{OR}=0,19 \sim \mathrm{IC}=0,05-0,69)$. A contracepção hormonal por outras vias (transdérmica ou transvaginal) foi também frequente $(21,7 \%)$ nesse grupo.

Em relação ao retorno para controle, 4,2\% $(\mathrm{n}=5)$ das pacientes do grupo privado não retornaram para avaliação do contraceptivo prescrito. No grupo da rede pública, 37,5\% $(n=45)$ das pacientes não retornaram para controle $(\mathrm{p}<0,05)$.

Quando se avaliou aderência ao tratamento prescrito, verificou-se que $92,2 \%$ das pacientes 
Tabela 1. Aspectos epidemiológicos e clínicos das pacientes estudadas.

\begin{tabular}{|c|c|c|c|c|c|c|}
\hline & \multicolumn{2}{|c|}{ Rede privada } & \multicolumn{2}{|c|}{ Rede pública } & \multirow[b]{2}{*}{$\chi^{2}$} & \multirow[b]{2}{*}{ p } \\
\hline & $\mathbf{N}$ & $\%$ & $\mathbf{N}$ & $\%$ & & \\
\hline \multicolumn{7}{|l|}{ Idade } \\
\hline 14-17 anos & 14,0 & 11,7 & 26,0 & 21,7 & 6,67 & 0,08 \\
\hline 18-24 anos & 52,0 & 43,3 & 53,0 & 44,2 & & \\
\hline 25-34 anos & 48,0 & 40,0 & 33,0 & 27,5 & & \\
\hline 35-42 anos & 6,0 & 5,0 & 8,0 & 6,7 & & \\
\hline \multicolumn{7}{|l|}{ Profissão } \\
\hline Do lar & 10,0 & 8,3 & 70,0 & 33,3 & 89,89 & 0,00 \\
\hline Estudante & 55,0 & 45,8 & 46,0 & 42,1 & & \\
\hline Fora do lar & 55,0 & 45,8 & 4,0 & 24,6 & & \\
\hline \multicolumn{7}{|l|}{ Vida sexual } \\
\hline Ativa & 98,0 & 81,7 & 100,0 & 83,3 & 0,03 & 0,43 \\
\hline Inativa & 22,0 & 18,3 & 20,0 & 16,7 & & \\
\hline \multicolumn{7}{|l|}{ Menarca } \\
\hline 09-14 anos & 114,0 & 95,0 & 102,0 & 85,0 & 5,6 & 0,01 \\
\hline 15-19 anos & 6,0 & 5,0 & 18,0 & 15,0 & & \\
\hline \multicolumn{7}{|c|}{ Tipo de ciclo menstrual } \\
\hline Irregular & 41,0 & 34,2 & 50,0 & 41,7 & 1,13 & 0,14 \\
\hline Regular & 79,0 & 65,8 & 70,0 & 58,3 & & \\
\hline \multicolumn{7}{|c|}{ Número de gestações } \\
\hline $00-00$ & 79,0 & 65,0 & 56,0 & 46,7 & 9,87 & 0,01 \\
\hline 01-01 & 27,0 & 22,5 & 36,0 & 30,0 & & \\
\hline $02-08$ & 14,0 & 11,7 & 28,0 & 23,3 & & \\
\hline \multicolumn{7}{|c|}{ Número de partos } \\
\hline $00-00$ & 86,0 & 71,7 & 60,0 & 50,0 & 14,28 & 0,01 \\
\hline 01-01 & 25,0 & 20,8 & 37,0 & 30,8 & & \\
\hline $02-07$ & 9,0 & 7,5 & 21,0 & 17,6 & & \\
\hline \multicolumn{7}{|c|}{ Número de abortos } \\
\hline $00-00$ & 107,0 & 89,2 & 109,0 & 90,8 & 1,41 & 0,49 \\
\hline 01-01 & 13,0 & 10,8 & 10,0 & 8,3 & & \\
\hline
\end{tabular}

da rede privada de saúde faziam uso da medicação prescrita anteriormente, mas 7,8\% $(n=9)$ não utilizaram a medicação e apontaram o desejo de engravidar $(n=4)$, supressão da lactação $(n=2)$, efeito colateral $(n=1)$ e dificuldade na aquisição da medicação $(n=2)$ como os responsáveis pela não aderência ao tratamento. No grupo público, $76 \%$ das pacientes faziam uso da medicação prescrita e $24 \%$ não utilizaram a medicação prescrita $(\mathrm{n}=18)$; e atribuíram ao desejo de engravidar $(n=6)$, simplesmente "não quiseram" $(n=5)$, efeito colateral $(n=4)$, esquecimento $(n=1)$, não conseguiu adquirir o medicamento $(n=1)$, outras $(\mathrm{n}=1)$, como os responsáveis pela não aderência ao tratamento $(\mathrm{p}=0,00 \sim \mathrm{IC}=0,11-0,64)$.

Não se verificaram diferenças em relação à frequência dos efeitos colaterais (cefaleia $(p=0,45)$, enjoo $(p=0,24)$, sangramento irregular $(p=0,62)$, mudança de humor $(p=0,22)$, sen- sibilidade nas mamas ( $p=0,22$ ), e não se evidenciaram efeitos colaterais maiores. Quando se avaliou a descontinuação da medicação, considerando as pacientes que apresentaram efeito colateral (setor privado, $n=16$; setor público, $n=8$ ), verificou-se que $6,3 \%$ das pacientes do setor privado suspenderam a medicação apenas pela presença desses efeitos. No grupo público, 50\% das pacientes suspenderam a medicação devido a estes efeitos ( $\mathrm{p}=0,03 \sim \mathrm{IC}=1,29-174,39)$.

No exame de controle, não se verificaram ganho de peso, alteração pressórica ou piora das manifestações androgênicas em ambos os grupos. Nos casos específicos de acnes, verificou-se que, das 27 pacientes do setor privado que apresentaram acnes no exame físico, 24 (88,8\%) apresentaram melhora clínica. No setor público, das sete pacientes nas quais se diagnosticou acne, a melhora clínica foi verificada em 42,8\% ( $p=0,01)$. Com- 
parando os outros sinais clínicos das manifestações androgênicas (aumento de pelos e seborreia), os resultados não foram estatisticamente significativos, embora 90,9\% e 75\% das pacientes do grupo privado tenham apresentado melhora da acne e seborreia, enquanto no grupo público essa melhora foi de $75 \%$ e $50 \%$, respectivamente.

\section{Discussão}

Em relação à idade, nossa amostragem foi de pacientes jovens. Como todas são candidatas à contracepção hormonal, não houve diferença estatística entre os grupos. Quando se avaliou o número de gestações e partos, verificou-se que as pacientes do setor público de saúde apresentaram maior número de gestações e partos. Esses resultados refletem o perfil social, já que a literatura afirma que pacientes com padrão socioeconômico mais baixo apresentam também maior número de gestações e partos no Brasil. Estudo realizado no município de Cachoeirinha, Porto Alegre, verificou que mulheres de baixo nível socioeconômico e escolaridade estagnada durante 25 anos demonstraram tendência à multiparidade, com pico percentual de aumento de dois para três filhos aos 24 anos $^{7}$. As mulheres do setor público são, em geral, donas de casa. Desse modo, a desigualdade social e econômica é apontada como fator de diferenciação das condições de saúde da população. Indicadores como escolaridade, renda e o local de moradia determinam tanto o acesso quanto a qualidade da assistência prestada a essas pacientes ${ }^{8}$.

Os exames de rotina, corrimento vaginal, contracepção, irregularidade menstrual, TPM e as manifestações androgênicas foram os mais frequentes. Tais resultados são compatíveis com os citados na literatura, já que alguns autores verificaram que os exames de rotina, corrimento vaginal e as alterações menstruais foram também os mais frequentes em suas séries de casos, merecendo atenção especial do médico no cuidado à saúde da mulher.

Estudo realizado na Faculdade de Medicina da Universidade Federal de Minas Gerais verificou que nas pacientes soropositivas para o vírus da imunodeficiência humana (HIV) essas queixas são frequentes durante o controle ginecológico ${ }^{9}$. Outro estudo realizado também na Faculdade de Medicina de Barbacena (MG) verificou que a irregularidade menstrual e os fluxos genitais patológicos são queixas comuns das pacientes durante o atendimento ginecológico ${ }^{10}$.
Entretanto, algumas queixas foram associadas às pacientes da rede privada de saúde, como por exemplo a tensão pré-menstrual (TPM) e manifestações androgênicas. Milhões de mulheres em idade reprodutiva apresentam sintomas emocionais, cognitivos e físicos relacionados ao seu ciclo menstrual. Elas demonstram irritabilidade intensa, frequentemente acompanhada de humor depressivo, assim como numerosas queixas mentais e somáticas. Tais sintomas são recorrentes durante a fase lútea do ciclo menstrual e interferem de maneira significativa no seu funcionamento social, ocupacional e sexual. O papel dos hormônios sexuais parece ter importância central, pois a síndrome pré-menstrual se agrava com o início dos fenômenos regressivos do corpo-lúteo, por volta do $24^{\circ}$ ou $25^{\circ}$ dia do ciclo. Entretanto, causas ambientais podem também estar relacionadas à TPM. Alguns alimentos foram associados ao seu desenvolvimento (chocolate, cafeína, sucos de frutas e álcool $)^{11-14}$. Estudo populacional realizado na Universidade Federal de Pelotas identificou que os sintomas da TPM foram mais prevalentes nos níveis econômicos elevados, na maior escolaridade, nas mais jovens e nas mulheres de cor branca, fato comparável a este estudo ${ }^{15}$.

Em relação às manifestações androgênicas, verificou-se maior associação com as pacientes da rede privada de saúde, já que $18,3 \%$ das pacientes da rede privada e 3,3\% das pacientes da rede pública apresentaram manifestação androgênica ao exame físico (acne, aumento de pelos e seborreia). A síndrome de ovários policísti$\cos (\mathrm{SOP})$ é doença endócrina complexa, que tem como elementos principais hiperandrogenismo e anovulação crônica. Caracteriza-se por irregularidade menstrual ou amenorreia e achados clínicos decorrentes do hiperandrogenismo (hirsutismo, acne, alopécia e seborreia). Representa uma das desordens endócrinas reprodutivas mais comuns em mulheres, acometendo em torno de $5 \%$ a $10 \%$ da população feminina em idade fértil ${ }^{16}$. Dado importante, entretanto, é a associação entre SOP com obesidade, pois cerca de $65 \%$ das mulheres com SOP têm o índice de massa corporal (IMC) acima de 27. A distribuição de gordura é frequentemente abdominal (visceral) e associada a anormalidades metabólicas (hipertensão, dislipidemia, resistência insulínica e intolerância à glicose). Como consequência, a circunferência abdominal está aumentada nesses casos (>88 $\mathrm{cm})$, assim como a relação cintura-quadril $(>0,85)$, parâmetros que estão associados a maior risco de doença cardiovascular. A obesidade 
também pode facilitar anormalidades metabólicas, como resistência à insulina. Em nosso estudo, a obesidade abdominal foi mais frequente nas pacientes da rede privada de saúde, o que sugere uma associação entre a obesidade abdominal e manifestação androgênica ${ }^{17}$.

Em relação aos outros dados do exame físico, não se verificou diferença significativa (exame de mamas, avaliação da pressão arterial, da genitália externa, trofismo vaginal e fluxo genital), até porque todas são jovens, sexualmente ativas, sem doença crônica preexistente, por isso não se esperam diferenças nessas distribuições.

As infecções genitais mais frequentes foram candidíase, tricomoníase e vaginose bacteriana, e conforme dados da literatura são realmente as principais infecções genitais. Estudo realizado na Faculdade de Medicina de Barbacena, com 252 pacientes, diagnosticou vaginose bacteriana em $12,5 \%$ dos casos, candidíase em $10,1 \%$ e tricomoníase em 1,8\%, dados comparáveis a esta avaliação ${ }^{11}$. Embora o fluxo genital patológico tenha sido motivador de consultas em 3,0\% das pacientes da rede pública, o exame ginecológico identificou o mesmo em $27,7 \%$ das pacientes. Acreditamos que essa diferença pode estar associada à percepção equivocada da paciente sobre o fluxo genital, desconhecimento sobre seu corpo, higiene inadequada e até a transitoriedade dos sintomas vaginais ${ }^{11,18-21}$.

As pacientes da rede privada de saúde beneficiaram-se do tratamento prescrito não apenas para a contracepção, já que ele foi prescrito também para o controle de manifestações androgênicas, o controle da dismenorreia e da TPM. Recente revisão sistemática da Cochrane verificou que os contraceptivos hormonais orais, quando comparados com o placebo, são eficazes na redução das acnes com processos inflamatórios associados ou não, sendo que o acetato de clormardinona e a ciproterona parecem ter efeito melhor sobre esse tratamento quando comparados ao levonogestrel e ao desogestrel ${ }^{22}$. Tais observações são compatíveis com nossos resultados, já que verificamos melhora clínica das acnes nas pacientes da rede privada de saúde $(p=0,01)$. Isso pode ser explicado porque as pacientes da rede pública utilizam compostos disponíveis, dificultando uma prescrição individualizada. Outro benefício adicional foi o controle da dismenorreia primária; segundo estudo realizado na Universidade de Wayne, em Detroit (EUA), a associação EE e desogestrel foi eficaz para reduzir as cólicas menstruais, como também o fluxo menstrual ${ }^{23}$. Tais resultados são também corroborados por outros estudos que sugerem que o tratamento da dismenorreia, quando não responde aos anti-inflamatórios convencionais, pode ser feito com contraceptivos combinados de estrogênio e progestágeno, embora haja necessidade de estudos randomizados com dosagens contraceptivas menores ${ }^{24-26}$.

No grupo privado, a dosagem hormonal de 15 ou 20 mcg de EE associados a gestodeno, levonogestrel ou desogestrel foi a mais frequentemente prescrita, objetivando a redução dos efeitos colaterais. Vários ensaios clínicos verificaram que contraceptivos hormonais orais com dosagens baixas de EE apresentam bom controle de peso corporal, baixa incidência de efeitos adversos, inclusive em pacientes acima de $35 \operatorname{anos}^{27,28}$. Revisão sistemática da Cochrane descreveu a segurança dos contraceptivos hormonais de baixa dose, embora a irregularidade menstrual seja maior nas usuárias dessas pílulas ${ }^{29}$. No grupo público, a dosagem mais prescrita foi a de $30 \mathrm{mcg}$ de EE associados ao levonogestrel, geralmente de distribuição gratuita nos postos de saúde. Entretanto, em nosso estudo, não houve diferença significativa na frequência dos efeitos colaterais, já que a maior frequência de prescrição em ambos os grupos foi de contraceptivos hormonais considerados de baixa dose ( $\leq 30 \mathrm{mcg}$ de $\mathrm{EE})$. A vantagem desses contraceptivos está na diminuição do risco venoso tromboembólico, da isquemia cardíaca e dos acidentes vasculares cerebrais (AVC), e sabese que dosagens abaixo de $30 \mu \mathrm{g}$ EE praticamente não alteram a coagulação. Acredita-se que o enfarto do miocárdio só ocorra em mulheres com mais de 35 anos ou em situações de múltiplos fatores de risco, como obesidade, tabagismo, hipertensão, hipercolestrolemia e diabetes ${ }^{30-32}$.

O retorno para controle ocorreu num intervalo de três a seis meses após a prescrição inicial da contracepção, e verificamos que as pacientes da rede privada de saúde retornaram para controle com maior frequência $(\mathrm{p}<0,05)$. Inquérito domiciliar no município de São Paulo (SP), com uma amostra representativa de 1.172 mulheres, selecionadas aleatoriamente, identificou que os principais motivos para a não realização da consulta ginecológica foram a ausência de problemas ginecológicos, vergonha, medo e dificuldades de acesso ${ }^{33}$. Outro estudo cita a má qualidade dos serviços de saúde, tempo de espera, problemas com agendamento, falta de médico ou greve como dificuldades que desestimulam o exame periódico ${ }^{34}$. Pode-se entender que uma adesão precária implica também pílulas esquecidas, ingestão sem respeitar a ordem e início de uma 
nova caixa antes ou depois do indicado. Entretanto, a falta de adesão ao contraceptivo hormonal, em especial ao oral, não é um problema específico do Brasil. Nos Estados Unidos, 20\% dos 3,5 milhões de casos de gravidez não desejadas estão relacionados com uma descontinuidade do método, sendo que outro estudo com mulheres europeias verificou que $10 \%$ delas haviam engravidado sem desejar e $18 \%$ eram usuárias de contraceptivo hormonal oral no momento em que ficaram grávidas. Alguns estudos descrevem que, nas classes sociais mais altas, cada mulher que está fazendo uso de anticoncepcional se esquece de tomar em média quatro pílulas por ano, enquanto nas classes sociais mais baixas o número é de 9,69 por ano ${ }^{35-37}$. Acreditamos que, em nosso estudo, a falta de aderência ao tratamento esteve associada ao nível cultural, menor no serviço público. A fim de evitar esses transtornos, temos implementado em nosso serviço palestras de apoio às pacientes e incremento das orientações durante a consulta médica, a fim de evitar que mitos possam prejudicar nosso acompanhamento.

Desse modo, conclui-se que dosagens contraceptivas menores ou iguais a $30 \mathrm{mcg}$ de etinilestradiol não determinam alterações na frequência dos efeitos colaterais. Entretanto, a aderência ao tratamento ainda é um grande problema para as usuárias do serviço público, o que pode estar associado a políticas públicas voltadas apenas à maternidade.

A promoção de uma vida sexual segura é uma meta do Ministério da Saúde. Acreditamos que, com incentivo, orientação e acesso à medicação, poderemos melhorar a aderência ao tratamento.

\section{Colaboradores}

PD Gomes, JB Zimmermmann, LMB Oliveira, KA Leal, ND Gomes, SM Goulart e DF Rezende participaram igualmente de todas as etapas de elaboração do artigo.

\section{Referências}

1. Pincus G. The control of fertility. New York: Academic Press; 1965.

2. Stadel BV. Oral contraceptives and cardiovascular disease. N Engl J Med 1981; 305:612-617.

3. Carr BR. Reevaluation of oral contraceptive classifications. Int J Fertil 1997; 42:133-144.

4. Carlborg L. Cyproterone acetate versus levonogertrel combined with ethinylestradiol in the treatment of acne. Acta Obstet Gynecol 1986; 134:29-32.

5. Camargos AF, Melo VH. Ginecologia ambulatorial. Belo Horizonte: Coopemed; 2001.

6. Organização Mundial da Saúde (OMS). Critérios de elegibilidade para uso de contraceptivos hormonais orais - Federação Brasileira de Ginecologia e Obstetrícia: manual de anticoncepção. [texto na Internet]. [acessado 2008 ago 29]. Disponível em: http://www. febrasgo.org.br

7. Nunes R, Galbinski A, Borges MB, Galbinski S, Cunha CN. Reprodução, anticoncepção e fatores socioculturais: uma análise. Reprodução 1988; 3(2): 98-101.

8. Leal MC, Gama SGN, Cunha CB. Desigualdades sociodemográficas e suas consequências sobre o peso do recém-nascido. Rev Saude Publica 2006; 40(3):466-473.

9. Zimmermmann JB, Melo VH, Castro LPF, Castilho DM. Associação entre a contagem de linfócitos TCD4+ e a gravidade da neoplasia intraepitelial cervical diagnosticada pela histopatologia em mulheres infectadas pelo HIV. Rev Bras Ginec Obstet 2006; 28:345-351.

10. Zimmermmann JB, Rezende DF, Nunes AA, Tourino AG, Almeida FC, Teixeira LMC, Moreira MC, Ferreira A. Aspectos epidemiológicos e clínicos de pacientes ginecológicas atendidas na Faculdade de Medicina de Barbacena. Revista Médica de Minas Gerais 2008; 18(3):160-166. 
11. Pires MLN, Calil HM. Associação entre transtorno disfórico pré-menstrual e transtornos depressivos. Rev Bras Psiquiatr 1999; 21(2):118-127.

12. Fernandes CE, Ferreira JAS, Azevedo LH, Pellini EAJ, Peixoto S. Síndrome da tensão pré-menstrual: o estado atual dos conhecimentos. Arq Méd ABC 2004; 29(2):77-81.

13. Cota AMM, Sousa EBA, Caetano JPJ, Santiago RC, Marinho RM. Tensão pré- menstrual. Femina 2003; 31(10):897-902.

14. Valadares GC, Ferreira LV, Corrêa Filho H, Romano Silva MA. Transtorno disfórico pré-menstrual: revisão, conceito, história, epidemiologia e etiologia. Rev Psiq Clin 2006; 33(3):117-123.

15. Silva CML, Gigante DP, Carret MLV, Fassa AG. Estudo populacional da síndrome pré-menstrual. Rev Saude Publica 2006; 40(1):47-56.

16. Junqueira PAA, Fonseca AM, Aldright JM. Síndrome dos ovários policísticos. Rev Assoc Med Bras 2003; 49(1):13-14.

17. Ferreira JA, Fernandes CE, Azevedo LE, Peixoto S. Síndrome da anovulação crônica hiperandrogênica e transtornos psíquicos. Rev Psiq Clin. [periódico na Internet]. 2006 [acessado 2008 set 8]; 33(3):145-151. Disponível em: http://www.hcnet.usp.br/ipq/revista/ vol33/n3/145.html

18. Eleutério Júnior J, Cavalcanti DIM. Contagem de morfotipos de Mobiluncus sp e concentração de leucócitos em esfregaços vaginais de pacientes com vaginose bacteriana. Rev Bras Ginec Obstet 2004; 26(3):221-225.

19. Giraldo PC, Amaral RLG, Gonçalves AK, Vicentini R, Martins CH, Giraldo H, Fachini AM. Influência da frequência de coitos vaginais e da prática de duchas higiênicas sobre o equilíbrio da microbiota vaginal. Rev Bras Ginec Obst 2005; 27(5):257-262.

20. Aleixo Neto A, Hamdam JS, Souza RC. Prevalence of candida in the vagina of women attended at a family planning service. Rev Bras Ginecol Obstet 1999; 21(8):441-445.

21. Maciel GP, Tasca T, De Carli GA. Aspectos clínicos, patogênese e diagnóstico de Trichomonas vaginalis. J Bras Patol Med Lab 2004; 40(3):152-160.

22. Arowojolu AO, Gallo MF, Grimes DA, Garner SE. Combined oral contraceptive pills for treatment of acne. In: The Cochrane Library. Oxford: Update Software; 2005. Issue 2.

23. Hendrix SL, Alexander NJ. Primary dysmenorrhea treatment with a desogestrelcontaining lowdose oral contraceptive. Contraception 2002; 66(6):393-399.

24. Dawood MY. Primary dysmenorrhea: advances in pathogenesis and management. Obstet Gynecol 2006; 108(2):428-441.

25. Harel Z. Dysmenorrhea in adolescents and young adults: etiology and management. J Pediatr Adolesc Gynecol 2006; 19(6):363-371.

26. Proctor ML, Roberts H, Farguhar CM. Combined oral contraceptive pill as treatment for primary dysmenorrhoea. In: The Cochrane Library. Oxford: Update Software; 2005. Issue 2.
27. Pereira Filho AS, Bedone AJ, Campos AA, Aleixo Neto A, Mello CR, Tomaz G, Barbosa IC, Leal JWB, Morais KM, Giordano M, Melo N, Mussiello R, Andrade RP, Souza RN, Formiga SMFN. Eficácia, tolerabilidade, controle de ciclo e bem-estar em mulheres acima de 35 anos utilizando um contraceptivo de baixa dose contendo gestodeno e etinilestradiol. Fol Méd 2000; 119(2):48-52.

28. Fernandes CE, Pereira Filho AS, Barbosa IC, Faggion D, Andrade RP. Avaliação da tolerabilidade, aceitabilidade e controle do ciclo em adolescentes usuárias de anticoncepcional hormonal combinado oral de baixa dose com gestodeno e etinilestradiol. Reprod Clin 2004; 19:12-17.

29. Gallo MF, Nanda K, Grimes DA, Schulz KF. Anticonceptivos orales combinados con $20 \mathrm{mcg}$ versus $>20$ mcg de estrógeno para la anticoncepción (Cochrane Review). In: La Biblioteca Cochrane Plus. Oxford: Update Software; 2007. Issue 1.

30. Kaiser E. Action of a new hormonal contraceptive. Geburst U Frauenheilkunde 1984; 44:651.

31. Hatcher RA, Rinehart W, Blackburn R, Geller JS, Shelton JD. Pontos essenciais da tecnologia da anticoncepção. Baltimore: Escola de Saúde Pública Johns Hopkins; 2001.

32. Schor N, Ferreira AF, Machado VL, França AP, Pirotta KCM, Alvarenga AT, Siqueira AAF. Mulher e anticoncepção: conhecimento e uso de métodos anticoncepcionais. Cad Saude Publica 2000; 16:377384.

33. Pinho AA, França Júnior I, Schraiber LB, D’Oliveira AFPL. Cobertura e motivos para a realização ou não do teste de Papanicolaou no município de São Paulo. Cad Saude Publica 2003; 19(2):303-313.

34. Brenna SMF, Hardy E, Zeferino LC, Namura I. Conhecimento, atitude e prática do exame de Papanicolaou em mulheres com câncer de colo uterino. Cad Saude Publica 2001; 17(4):909-914.

35. Rosenberg M, Waugh MS. Causes and consequences of oral contraceptive noncompliance. $\mathrm{Am} \mathrm{J} \mathrm{Ob-}$ stet Gynecol 1999; 180(2):27-69.

36. Serfaty D. Medical aspects of oral contraceptive discontinuation. Adv Contracept 1992; 8(1):21-33.

37. Rosenberg MJ, Waugh MS, Burnhill MS. Compliance, counseling and satisfaction with oral contraceptives: a prospective evaluation. Fam Plann Perspect 1998; 30(2):89-92.

Artigo apresentado em 08/08/2008 Aprovado em 15/01/2009

Versão final apresentada em 20/02/2009 